\title{
Evaluación de Bancos Naturales de Macha Mesodesma donacium Asentadas en Playa Santa Rosa con la Finalidad de Repoblar Áreas Aledañas del Litoral
}

\author{
Assessment of Natural Banks Macha Mesodesma donacium \\ seating on Santa Rosa Beach in order to repopulate the \\ Surrounding Areas Coast \\ 'Gregoria Teresa Castillo Medina
}

\begin{abstract}
RESUMEN:
El presente documento contiene los resultados de las evaluaciones del banco natural del recurso macha en playa Santa Rosa efectuadas en el año 2009. orientado a estimar: parámetros poblacionales de Mesodesma donacium, condiciones bio-pesqueras y ecológicas; que servirán de base para la elaboración de un área de repoblamiento o Area de manejo. Estas normas de regulación son los úmicos instrumentos que garantizan el control de la sustentabilidad del recurso y permite tomar medidas de ordenamiento pesquero asegurando la conservación.
\end{abstract}

Palabras clave: biomasa, Mesodesma donacium, repoblamiento

ABSTRACT:

This document contains the results of evaluations of resource natural bank of macha on Santa Rosa Beach applied in 2009, aimed to estimate population parameters of $M$. donacium, ecological and bio-fishing condirions, which will serve as the basis for the elaboration of a stocking or management area. These regulatory standards are the only instruments that guarantee the control of the resource sustainability and permit to ensure to the conservation of fisheries management.

Keywords: biomass, Mesodesmadonacium, repopulation

'Master of Sciencie con Mencion en Gestion Ambiental y Desarrollo Sostenido, Ingeniero Pesquero. Facultad de Ciencias Agropecuarias.

Universidad Nacional Jorge Basadre Grohmann 


\section{INTRODUCCIÓN}

Por diversas causas, las pesquerías de recursos bentónicos en Perú han mostrado grandes fluctuaciones en el tiempo y en el espacio, generando en el mediano plazo alteraciones y, normalmente, declinaciones que producen inestabilidad en la cadena de comercialización de los recursos y consecuentemente, deterioro en las condiciones socioeconómicas del sector pesquero artesanal. Si bien, la causa principal de estas fluctuaciones se ha atribuido a la explotación pesquera, también existe evidencia del impacto de causas naturales sobre la abundancia y distribución de las poblaciones bentónicas sometidas a explotación, tales como la contaminación, el fenómeno «El Niño» y movimientos sísmicos, entre otros.

Las comunidades de Pescadores Artesanales de las Playas; Santa Rosa, Los Palos, La Yarada, Llostay y Cánepa, quienes se han dedicado principalmente durante muchos años a la recolección de machas, a partir del año 1999 se han visto seriamente afectadas en su actividad económica, a causa de que dicho recurso pesquero ha sido reducido a volúmenes ínfimos por varios factores naturales y humanos.

Uno de los factores se atribuye a que desde el año 1992 se ejerció una fuerte presión extractiva del recurso macha debido a la gran demanda solicitada por comerciantes y empresarios pesqueros de la localidad de Tacna, esta situación indujo a la reducción de la biomasa poblacional de la especie. Posteriormente otro de los acontecimientos fue la presencia del fenómeno "El niño 97-98", que provocó durante el verano del '98 la mortalidad masiva y paulatina de las poblaciones de Mesodesma donacium en los bancos naturales del sur del Perú; como ser: Lomas, Camana, Mejía, Boquerón, La Punta, Corío, Pocoma y Pozo de Lizas.

En prospecciones oceanográficas de recursos bentónicos realizados en el año 2006, a cargo de la Dirección Regional de la Producción-Tacna y el Instituto del Mar del Perú, se han identificado desde la Playa Santa Rosa hasta Playa Los Palos tres núcleos de bancos naturales de macha. Ante la necesidad de contribuir al Ordenamiento Pesquero, se requiere de un diagnóstico de los bancos naturales que incluya la ubicación, caracterización y clasificación de los bancos de acuerdo a criterios biológico-pesqueros, ecológicos y socio-económicos para tomar acciones futuras.

En este contexto, la explotación de la macha implicará un manejo en el cual, sobre información fundada, se establecerán normas de regulación que promuevan una serie de medidas, orientadas a una administración sostenible del recurso en el sector señalado.

No obstante, para que exista actividades de repoblamiento de este recurso se requiere de semilla y/o reproductores, teniendo en cuenta que en la actualidad no se registran Estudios de Línea Base en cuanto a stocks poblacionales de macha que puedan utilizarse como fracción donante hacia playas aledañas que fueron eminentemente macheras.

\section{MATERIALES Y MÉTODOS}

\subsection{Georeferenciación del Área de Estudio}

Para la georeferenciación del borde costero se utilizó un GPS GARMIN $76 \mathrm{~S}$, se georeferenciaron puntos en coordenadas geográficas en el Datum WGS-84 en la línea de máxima marea cada 10 a 50 metros de distancia, los datos obtenidos se han pasado a una planilla de datos Excel $\mathrm{Xp}$, estos han sido transferido a programas de mapeo de MAIP INFO 7.5 y SURFER 8.0. (Figura 1).

\subsection{Técnicas y métodos de recolección de datos}

La unidad de muestreo que se utilizó fue el cuadrante de $0.25 \mathrm{~m}^{2}$ (Pringle, 1997). El mencionado autor ha demostrado que en la evaluación de recursos bentónicos, un cuadrante de $0.5 \mathrm{~m} \times 0.5 \mathrm{~m}$, permite obtener un error de muestreo satisfactorio en concordancia con la facilitación del recuento de ejemplares y maximización de la efíciencia de muestreo.

Las actividades relacionadas con el recuento de los individuos de cada cuadrante y la recolección de ejemplares para la determinación de la estructura de tamaños poblacional serán realizadas por pescadores macheros. Atendiendo a las particularidades del oleaje, la energia del ambiente de esta zona y considerando como un factor importante la experiencia de los pescadores macheros, quienes realmente poseen conocimiento de los sectores a evaluar y poseen la rapidez necesaria para la extracción del recurso, asegurando además exactitud en la ubicación de los ejemplares dentro del área de muestreo.

El muestreo en terreno contempló la realización 10 transectas separadas cada 100 metros, cubriendo las áreas de donde habita el recurso, la evaluación directa de la zona. En cada transecta se utilizo un número de 3 estaciones de muestreo (alturas de: rodilla, cintura y pecho) Figura 2. Cada uno de los cuadrantes dispuestos fueron georeferenciados mediante la utilización de un GPS marca GARMIN, modelo ETREX LEGEND (5 m de precisión). En cada cuadrante se registró la composición del sustrato (fango, arena fina, arena gruesa, y gravilla), la abundancia machas adultas y semillas de machas, adicionalmente, se selecciono una muestra representativa de ejemplares por cuadrante, los cuales fueron colocadas en bolsas etiquetadas para el muestreo biológico posterior.

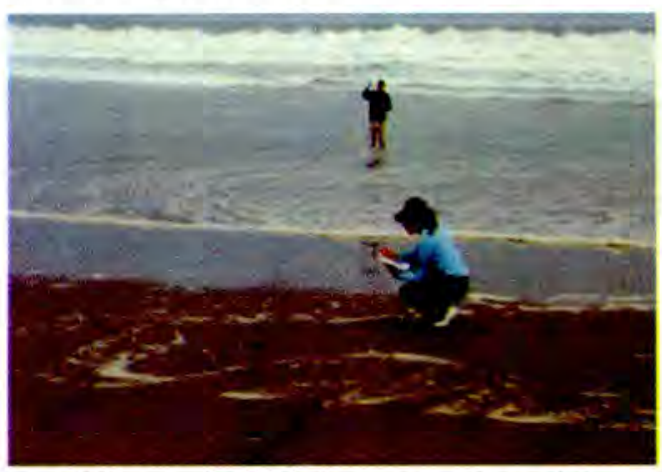

Figura $\mathrm{N}^{\circ} 01$ : Registro de datos georeferenciales en una Transecta mediante GPS 


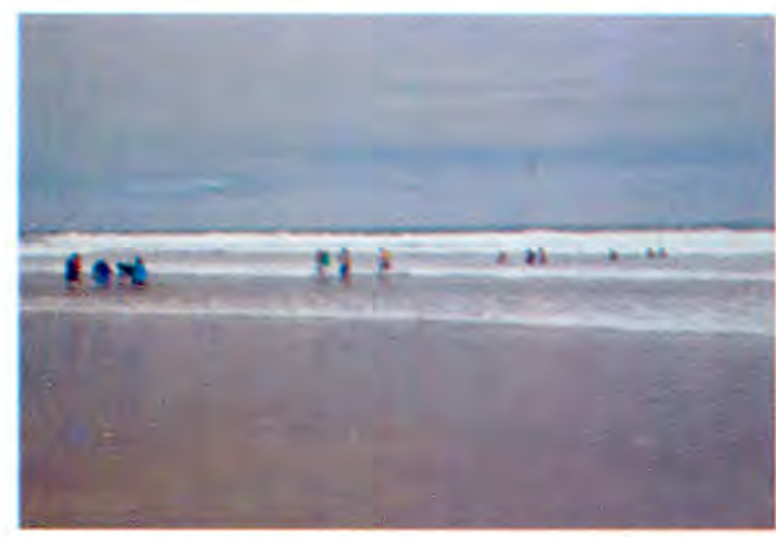

Figura $\mathrm{N}^{\circ} 02$ : Muestreo de machas según la altura de agua en una transecta.

\section{RESULTADOS}

\subsection{Características del área de estudio}

La Figura 3 presenta un mapa el cual nos permite ubicar geográficamente el área en estudio, el mismo que tiene una extensión de $1 \mathrm{~km}$ de playa; y donde el recurso se encuentra distribuido en un cinturón cuyo ancho es de 86 metros, formando un rectángulo cuyos vértices se muestra en dicha figura (A-B y C-D), el total el área de estudio es de 8.83 hectáreas.

\subsection{Distribución del Recurso}

Se ha identificado un parche muy abundante con una concentración de densidad que oscilan desde 20 a 30 ind $/ \mathrm{m}^{2}$ en dicho parche y en cuyo interior se muestra dos núcleos de alta concentración: el primero y el más grande se encuentra al frente del poblado de Santa Rosa con una concentración que está comprendida de 30 a 50 ind $/ \mathrm{m}^{2}$ y el segundo de menor dispersión, que está ubicado al sur de la playa Santa Rosa. En general la macha Mesodesma donacium se distribuye latitudinalmente mediante parches con variaciones espaciales de concentración y a la vez este recurso distribuye longitudinalmente a tres alturas de profundidad $(0.50,1.10$ y 1.6 metros $)$ como se ilustra en la Figura 2.

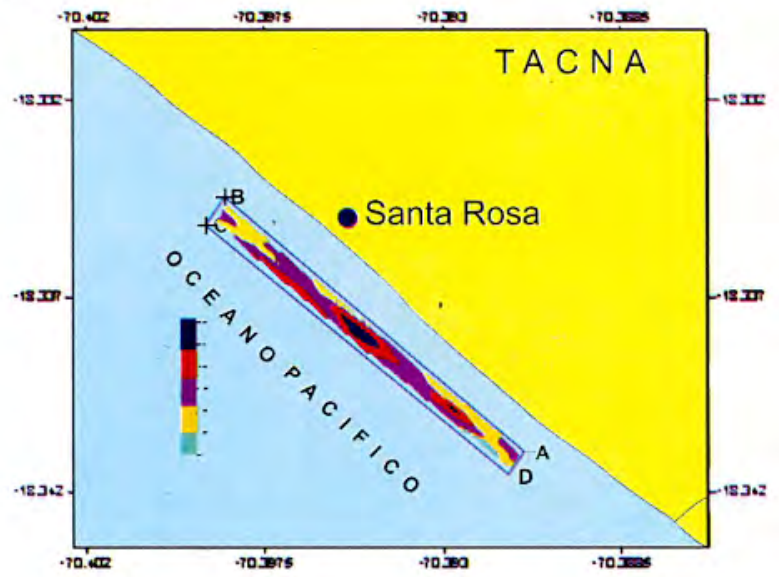

Figura $\mathrm{N}^{\circ} \mathbf{0 3}$ : Distribución y concentración del recurso macha en Playa Santa Rosa

\subsection{Comunidad Bentónica}

Composición Especiológica de la macro infauna intermareal

En un total de 6315 individuos recolectados en la zona Intermareal del área de estudio se identificaron 9 especies, agrupándose en 3 Phyllum.

\begin{tabular}{|c|c|c|}
\hline ESPECIES & $\begin{array}{l}\text { NOMBRE } \\
\text { CIENTIFICO }\end{array}$ & $\begin{array}{l}\text { NOMBRE } \\
\text { COMUN }\end{array}$ \\
\hline \multirow[t]{2}{*}{ MOLLUSCA } & Mesodesma donacium m & Macha \\
\hline & Mulinia edulis & Almejita \\
\hline \multirow[t]{2}{*}{ POLYCHAETA } & Nephtys sp & Poliqueto \\
\hline & Nematomorpha & Poliqueto \\
\hline \multirow[t]{5}{*}{ CRUSTACEA } & Emerita andiloga & muy muy \\
\hline & Isocheles pacificus & cangrejo ermitaño \\
\hline & Pseudocorystes sicarius & cangrejo ovalado \\
\hline & Lepidopa chilensis & muy muy blanco \\
\hline & Bellia picta & cangrejo plano \\
\hline
\end{tabular}

(a)

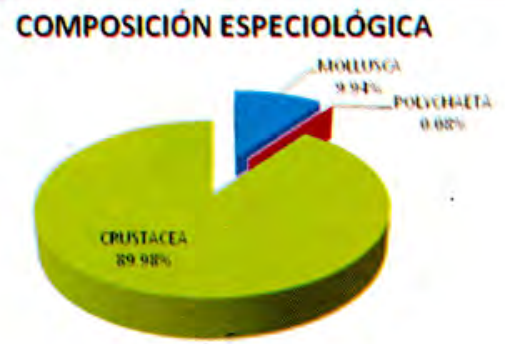

(b)

Figura $\mathbf{N}^{\circ}$ 04: (a) Diversidad por phyllum, (b) diversidad relativa (\%) por phyllum de la Playa Santa Rosa

En la figura 04 (a), se muestra que el $89.98 \%$ corresponde a crustáceos, seguido de $9.94 \%$ de moluscos y $0.08 \%$ de poliquetos.

\section{Abundancia de Especies (riqueza especifica)}

El recurso con mayor abundancia fue el muy muy Emerita análoga $(88.57 \%)$, seguido de macha Mesodesma donacium (5.92\%), almejita Mulinia edulis $(4.02 \%)$, Cangrejo ermitaño Isocheles pacificus $(1.22 \%)$ y el resto de los recursos cada uno no supera el $(0.08 \%)$. Así como se muestra en la Figura 5.

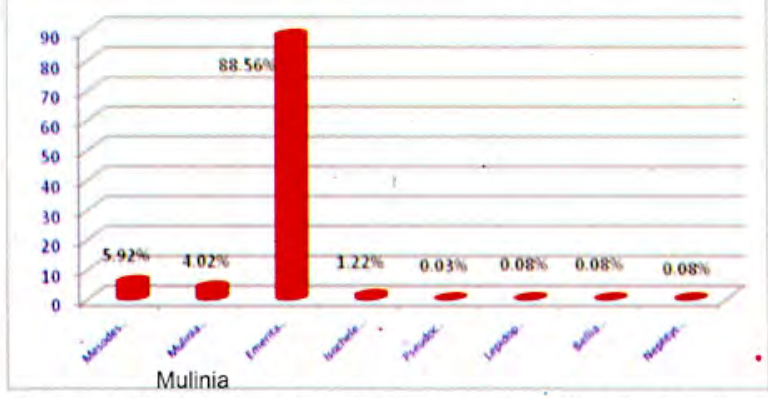

Figura $\mathbf{N}^{\circ} 05$ : Abundancia de especies (\%) de la playa Santa Rosa 


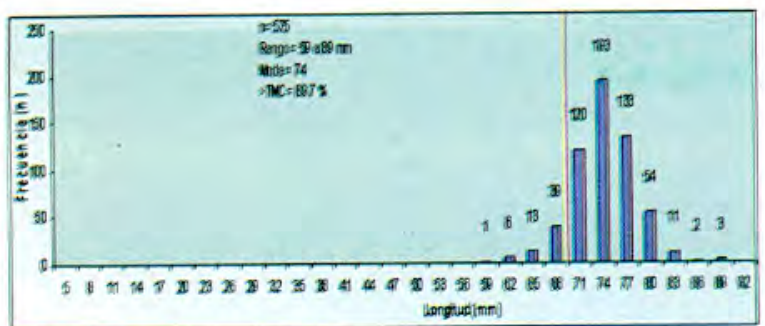

Figura No06: Estructura de tallas del recurso macha Mesodesma donacium de playa Santa Rosa

En $33.6 \%$ de la población de las muestras es de $74 \mathrm{~mm}$ de longitud, seguido de $23.1 \%$ son de $77 \mathrm{~mm}$ de longitud, en general la población está conformado por individuos adultos (Tabla 1)

Tabla $\mathbf{N}^{\circ} 01$ : Agrupación de los intervalos de clase y frecuencia de tallas

\begin{tabular}{cccc}
\hline $\begin{array}{c}\text { Intervalo } \\
\text { de clase }\end{array}$ & $\begin{array}{c}\text { Frecuencia } \\
\text { (n) }\end{array}$ & $\begin{array}{c}\text { Frecuencia } \\
(\%)\end{array}$ & $\begin{array}{c}\text { Frecuencia } \\
\text { Acumulada } \\
(\%)\end{array}$ \\
\hline 59 & 1 & $0.2 \%$ & $0.2 \%$ \\
62 & 6 & $1.0 \%$ & $1.2 \%$ \\
65 & 13 & $2.3 \%$ & $3.5 \%$ \\
68 & 39 & $6.8 \%$ & $10.3 \%$ \\
71 & 120 & $20.9 \%$ & $31.1 \%$ \\
74 & 193 & $33.6 \%$ & $64.7 \%$ \\
77 & 133 & $23.1 \%$ & $87.8 \%$ \\
80 & 54 & $9.4 \%$ & $97.2 \%$ \\
83 & 11 & $1.9 \%$ & $99.1 \%$ \\
86 & 2 & $0.3 \%$ & $99.5 \%$ \\
89 & 3 & $0.5 \%$ & $100.0 \%$ \\
& 575 & $100.0 \%$ & \\
\hline
\end{tabular}

\section{Relación Longitud Peso}

La ecuación que mejor ajusta a los datos biométricos obtenidos es la potencial, donde se muestra que el crecimiento es de tipo alométrico con un coeficiente de correlación de r2=0.54 de una muestra de 575 ejemplares de macha Figura 7.

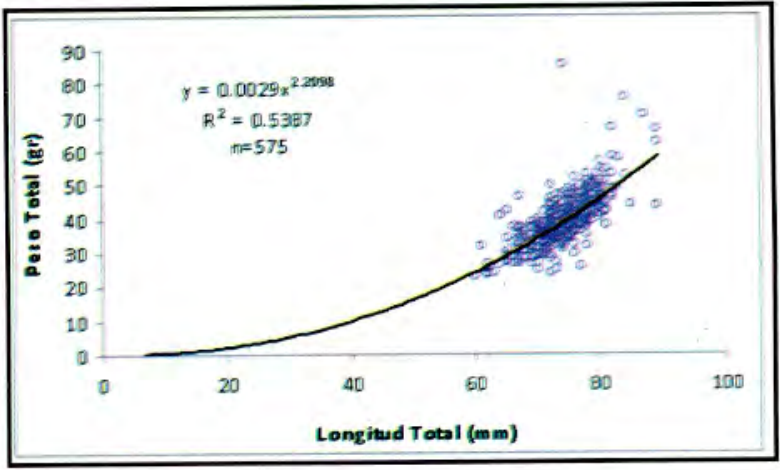

Figura $\mathbf{N}^{\circ} 07$ : Relación longitud y peso de Mesodesma donacium de playa Santa Rosa

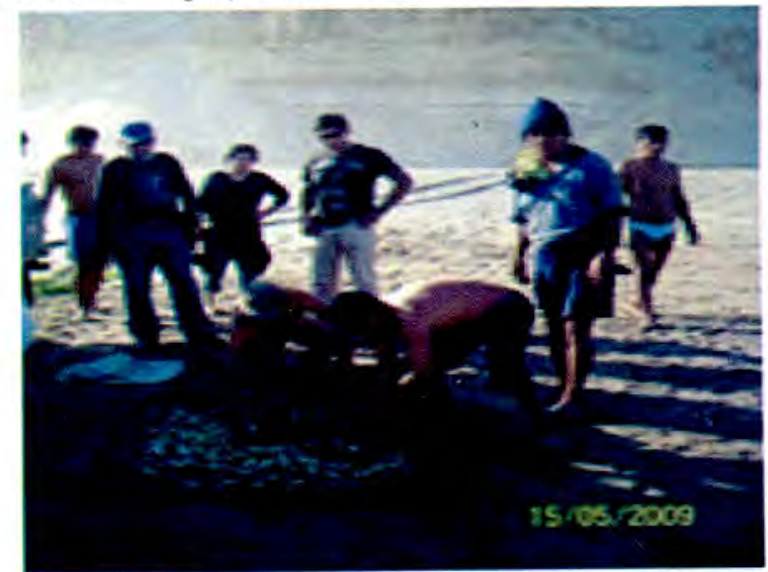

Figura $\mathrm{N}^{\circ} 08$ : Ejemplares reproductores de ${ }^{`}$ Macha muestreados en zona de estudio

Tabla N02: Abundancia y biomasa de ejemplares del recurso Macha en playa Santa Rosa-2009

\begin{tabular}{|c|c|c|c|c|c|c|c|}
\hline Condiciones Biopesqueras & Unidades & & & tos de Densid & & & \\
\hline Estrato & & I & II & III & IV & V & TOTAL \\
\hline Área $\left(\mathrm{m}^{2}\right)$ & $\mathrm{m}^{2}$ & 1349 & 13296.3 & 31129.7 & 10840.4 & 3744.6 & 60360 \\
\hline Densidad media del estrato & Individuos & 7.0 & 16.9 & 25.2 & 30.3 & 51.0 & \\
\hline Biomasa media del estrato & g & 292.5 & 677.0 & 969.4 & 1280.7 & 1997.7 & \\
\hline Varianza de la densidad del estrato & > & 13.5 & 6.7 & 11.0 & 69.3 & 50.0 & \\
\hline Varianza de la biomasa del estrato & & 23200.7 & 13190.5 & 13512.4 & 18681.8 & 6463.8 & \\
\hline Varianza de la densidad media & & 2.7 & 0.6 & 1.8 & 23.1 & 25.0 & \\
\hline Varianza de la biomasa media & & 4640.1 & 1199.1 & 2252.1 & 6227.3 & 3231.9 & \\
\hline DENSIDAD TOTAL (individuos) & individuos & 9443.0 & 224828.3 & 783430.8 & 328825.5 & 190974.6 & 1537502.2 \\
\hline BIOMASA TOTAL (gramos) & $\mathrm{g}$ & 394582.5 & 9001595.1 & 30177650.0 & 13882938.9 & 7480400.2 & 60937166.7 \\
\hline Densidad Media Estratificada & ejem $/ m^{2}$ & 25.47 & ejem $/ \mathrm{m}^{2}$ & & & & \\
\hline Biomasa Media Estratificada & $\mathrm{g} / \mathrm{m}^{2}$ & 1009.56 & $\mathrm{gr} / \mathrm{m}^{2}$ & 1.00956207 & $\mathrm{~kg} / \mathrm{m}^{2}$ & & \\
\hline POBLACION TOTAL & ejemplares & 1537502.2 & ejemplares & & & & \\
\hline BIOMASA TOTAL & $\mathrm{g}$ & 60937166.7 & gramos & 60937 & kilogramos & 60.9 & toneladas \\
\hline $\begin{array}{l}\text { Varianza de la Densidad Media } \\
\text { Estratificada }\end{array}$ & & 1.36 & & & & & \\
\hline $\begin{array}{l}\text { Varianza de la Biomasa Media } \\
\text { Estratificada }\end{array}$ & & 872.81 & - & & & & \\
\hline Limite Confianza Densidad & & 25.47 & $+/-$ & 2.85 & 11.20 & $\%$ & \\
\hline Limite Confianza Biomasa & & 1009.56 & $+1-$ & 72.29 & 7.16 & $\%$ & \\
\hline
\end{tabular}

\section{LEYENDA:}

Estrato I:

Estrato II:

Estrato III: densidades de 0 a 10 machas $/ \mathrm{m} 2$ densidades de 20 a 30 machas $/ \mathrm{m} 2$ densidades de 40 a 60 machas $/ \mathrm{m} 2$
Estrato IV:

Estrato V: densidades de 10 a 20 machas $/ \mathrm{m} 2$ densidades de 30 a 40 machas / $\mathrm{m} 2$ 


\subsection{Abundancia y Biomasa}

El área de estudio comprende $1 \mathrm{~km}$ de playa, en un cinturón de 86 metros lo que representa 8.83 hectáreas. el área habitable del recurso macha es de solo 6.036 hectáreas de los cuales se ha determinado las áreas de distribución según los estratos de densidad; en la cual la mayor parte de área presenta una distribución con una densidad media de 25.2 ejemplares $/ \mathrm{m}^{2}$ comprendidos en 3.1 ha (estrato III), seguido de 16.9 ejemplares $/ \mathrm{m}^{2}$ alcanzando una área de 1.3 ha (estrato II), seguido de 30.3 ejemplares $/ \mathrm{m}^{2}$ abarcando un área de 1.08 ha (estrato IV), 51 ejemplares $/ \mathrm{m}^{2}$ comprendido en un área de 0.37 ha (estrato $V$ ) y 7 ejemplares $/ \mathrm{m}^{2}$ abarcando una área de 0.13 ha (estratol).

La abundancia poblacional del área de estudio es de I 537502 individuos de macha, las estimaciones por cada estrato se presenta en la Tabla I en cuyos resultados se observa que el estrato III aporta la mayor cantidad de individuos de macha.

\section{DISCUSIÓN}

La playa Santa Rosa contempló la realización de 30 estaciones de muestreo, donde se han colectado 575 individuos de macha cuya estructura de tallas está comprendida entre 59 a $89 \mathrm{~mm}$ de longitud medida desde el borde anterior hasta el borde posterior, observándose una moda de $74 \mathrm{~mm}$ y además presenta una agrupación normal.

Los individuos mas grandes se localizan en la zona sur y a medida que se avanza hacia el norte los individuos vienen disminuyendo gradualmente su longitud. el $89.7 \%$ de los ejemplares se encuentran superior a la talla comercial quien a la vez supone que ya han desovado al menos una vez. Sin embargo es claramente notorio que no se ha tenido ningún registro de individuos de semilla ni juveniles, lo que pone en serio peligro su recuperación natural del recurso macha. Es por ello que es altamente viable que se implemente el área repoblamiento.

El presente estudio muestra información téenica donde en ninguna de las estaciones de muestreo se obtiene individuos reclutas ni juveniles, lo que hace notar que existe alto riesgo de colapsar nuevamente el recurso macha, ya que los individuos evaluados su población está conformado por ejemplares solo adultos, ante una eventual extracción podría llevar nuevamente a la desaparición de este recurso.

El recurso vive enterrado en arena fina de preferencia menor a 300 micras y distribuida entre 5 a $20 \mathrm{~cm}$ de profundidad. La distribución y concentración del recurso en el área de estudio no es uniforme, el recurso forman parchosidad con diferentes densidades.

El área de muestreo está doninada por ejemplares de muy muy (Emerita análoga), bajo este contexto donde existe mayor abundancia de este crustáceo menor es la diversidad.

\section{CONCLUSIONES}

1. La población de macha en las playas Santa Rosa, está conformado por solo individuos adultos comprendidos en un intervalo de clase de 59 a 89 min de longitud total.

2. En el área de estudio se encuentra una población de 1.5 millones de ejemplares de macha y 60.9 toneladas de biomasa de Mesodesma donacium.

3. El $89.7 \%$ de la población está por encima de la talla comercial por ende es la población en condiciones para su explotación.

4. Esta playa se presenta completamente desprotegida y actualmente la accesibilidad es alta, susceptible a que pescadores furtivos, y veraneantes extraigan el recurso sin ningún criterio técnico, más aun que la población de macha que se encuentra en playa Santa Rosa esta en desequilibrio poblacional puesto que no se cuenta con individuos reclutas que aseguren su sostenibilidad, por to que es necesario que se implementen los áreas de Repoblamiento y que su vigilancia y manejo este a cargo de las organizaciones sociales de pescadores de orilla.

\section{REFERENCIAS BIBLIOGRÁFICAS}

Aburto, James. Estudio Biológico Pesquero al Recurso Macha de la X Región. Proyecto FIP - $\mathrm{n}^{\circ} 2000-17$. Informe Final. Universidad Católica del Norte. Chile. $2001.237 p$.

Alarcon, Alí. Recuperación de la Biodiversidad de los Recursos Bentónicos de la Caleta Maintencillo. Mediante el Redoblamiento Integrado y Manejo Sustentable de Especies con algún Grado de Vulnerabilidad. Instituto de Fomento Pesquero. Informe Final. Chile, 2001.84 p.

Ariz, Luis. Bases para el Ordenamiento y Desarrollo de las Pesquerias Artesanales del Recurso Macha (Mesodesma donacium) en Chile Central.. Informe Final. $1^{\circ}$ Ed. Instituto de Fomento Pesquero. Chile, 1999.61 p.

Caddy, Fernando. Recursos Marinos Vivos y su Desarrollo Sostenible, Perspectivas Institucionales y Medioambientales. FAO. Documento Técnico de Pesca $\mathrm{N}^{\circ}$ 353. Roma, 1996.191 p.

Cardenas, Fernando. Entrenamiento para Pescadores Artesanales Acuicultores de X Región Los Lagos. $1^{\circ}$ Ed. Informe Final. Chile, 2001.94 p.

Dadon. José. Evaluación del Estado de la Almeja Amarilla (Mesodesma mactroides) en el Partido de la Costa durante el año 2001. Facultad de Ciencias Exactas y Naturales. Universidad de Buenos Aires- Argentina, 2002. 25 .

Hurtado, Antonio. Desarrollo Comunitario y Transferencia Tecnológica en la Costa Sur de Iquique. 
Caleta Los Verdes. Región de Tarapacá. $1^{\circ}$ Ed. Universidad Arturo Prat. Chile, 2000. $60 \mathrm{p}$.

Jaramillo, Eduardo. Ordenamiento de la Pesquería de Machas en la VIIII Región. Informe Final Proyecto FIP 2003-17Universidad católica del Norte. Chile $212 \mathrm{p}$.

Jerez, Gerardo. La Pesquería de la Macha Mesodesma donacium :Molusco, Bivalvia del Área de Manejo de Bahía Los Choros, IV Región. Un caso de Evaluación, Manejo y Co-administración Oportuna. $1^{\circ} \mathrm{Ed}$. XX Congreso Ciencias del Mar. Coquimbo-Chile, 2000. 28 p.

Ministerio de la Producción. Plan Nacional para el Desarrollo de la Pesca Artesanal. Lima - Perú, 2002. $85 p$.

Rees, Angel. Estudio Biológico Pesquero del Recurso Macha en la X Región. Informe Final. Instituto de Fomento Pesquero. División de Evaluación de Pesquerias Nacionales. Chile, 2001.242 p.
Saavedra, Fernando. Manejo Sostenible del Recurso Macha (Mesodesma donacium) puerto de lquique. $2^{\circ}$ Ed. Informe Final. Departamento de Investigaciones Marinas. Universidad del Norte de Chile, 1990.73 p.

Segovia, Ernesto. Estudio de Situación Base para el Área de Manejo de Recursos Bentónicos de Caleta Caramucho, Informe Final Subpesca, Chile, 2002.35 p.

Sotz, Walter. Distribución de Juveniles Recientemente Asentados de Mesodesma donacium (Lamarck, 1818) Molusco Bivalvo en Tres Bahias de la Cuarta Región. $1^{\circ}$ Ed. Investigaciones Pesqueras. Coquimbo-Chile, 1996. $40 \mathrm{p}$.

\section{Correspondencia:}

Gregoria Teresa Castillo Medina

Citdad Universitaria Fundo "Los Granados"

Calle Miraflores s $/ \mathrm{n}$. Tacna. Perú

gcastillom@unjbg.edu.pe 